\title{
Le séminaire européen d'ethnomusicologie, sienne, août 1989
}

\section{Peter Crowe}

Traducteur : Isabelle Schulte-Tenckhoff

\section{OpenEdition \\ Journals}

Édition électronique

URL : http://journals.openedition.org/ethnomusicologie/2416

ISSN : 2235-7688

Éditeur

ADEM - Ateliers d'ethnomusicologie

Édition imprimée

Date de publication : 1 janvier 1990

Pagination : 261-267

ISBN : 2-8257-0423-7

ISSN : $1662-372 X$

Référence électronique

Peter Crowe, «Le séminaire européen d'ethnomusicologie, sienne, août 1989 », Cahiers

d'ethnomusicologie [En ligne], 3 I 1990, mis en ligne le 15 octobre 2011, consulté le 20 avril 2019. URL http://journals.openedition.org/ethnomusicologie/2416 


\section{RENCONTRES}

\section{LE SÉMINAIRE EUROPÉEN D'ETHNOMUSICOLOGIE, SIENNE, AOÛT 1989*}

La sixième réunion annuelle du Séminaire européen d'ethnomusicologie (SEEM) fut organisée du 18 au 21 août 1989 à Sienne par les membres italiens du SEEM en collaboration avec les Séminaires internationaux d'ethnomusicologie de l'Accademia Musicale Chigiana. Le SEEM se compose d'ethnomusicologues professionnels et d'étudiants avancés vivant et travaillant en Europe (ou ayant effectué la plus grande partie de leurs études en Europe), qui se réunissent chaque année pour échanger opinions et résultats de recherche et discuter de problèmes communs. Cependant, les domaines de spécialisation des membres ne se limitent pas à l'Europe, mais couvrent le monde entier. Depuis la fondation du Séminaire en 1982, son fonctionnement a surtout été assuré par John Blacking (Belfast). A partir d'une poignée de membres fondateurs, le nombre d'adhérents s'est accru à cent en 1986 et à près de deux cents en 1989 (y compris les étudiants). Les conférences précédentes eurent lieu à Cologne (1983), Belfast (1985), Londres (1986), Paris (1987) et Tuczno/Pologne (1988). La septième rencontre est prévue pour octobre 1990 à Berlin, et des offres ont été reçus de Moscou et de Genève pour les années suivantes. Les actes du congrès de Londres ont été publiés (Philipp Ed. 1989), mais le SEEM n'a pas encore élaboré une politique de publication qui lui soit propre.

Le SEEM est devenu un événement majeur pour les ethnomusicologues européens, bien qu'il manque encore de représentativité (pour l'instant, il n'y a pas d'adhérents en Espagne et en Grèce). Sa publication interne intitulée Info, à parution intermittente, en est à son quinzième numéro ${ }^{1}$.

\footnotetext{
Traduit de l'anglais par Isabelle Schulte-Tenckhoff.
}

Info s'est borné jusqu'ici à servir de bulletin d'information. Son rédacteur est Francis Cameron, 12 Norreys Avenue, Oxford OX1 4SS, Royaume-Uni, tél. (865) 24 0058. Pour tout renseignement utile au sujet du SEEM (notamment en ce qui concerne l'adhésion), écrire à: European Seminar in Ethnomusicology, Business Office, B.P. 1, Av. de la Poste, F-31150 Fenouillet, France. 
Voilà la structure fondamentale d'une association qui fournit maintenant un apport majeur à l'ethnomusicologie et qui ne tardera pas à être connue de nombreux lecteurs européens des Cahiers de musiques traditionnelles. Si le présent compte rendu vise, entre autres, à mieux faire connaître le Séminaire ailleurs et à établir des liens plus étroits avec les Cahiers, son objectif principal est de donner un aperçu tout à fait personnel de la rencontre de Sienne.

D'autres auraient peut-être été mieux qualifiés que moi pour rédiger ce compte rendu; mais il se trouve que j'ai enregistré sur cassettes près de dixhuit heures (sur vingt) de la réunion et que j'ai tout naturellement eu l'occasion d'étudier en détail certaines interventions et de suivre de près le déroulement de nombreux débats.

Le plus souvent, ces derniers sont les produits les plus intéressants mais aussi les plus éphémères d'une conférence. Mes enregistrements ne portent que sur les réunions formelles, alors que les nombreuses discussions informelles lors des repas notamment, si fascinantes fussent-elles, ne peuvent être restituées que de mémoire et par ouï-dire. J'ai aussi été en mesure de comparer la réunion de Sienne avec celle de Belfast de 1985, à laquelle j'avais également participé.

Les langues de travail furent, à part égale, le français et l'anglais; on entendit aussi un peu d'allemand, et la plupart des participants venus d'Union soviétique eurent besoin d'interprètes, même lors des rencontres informelles.

A la question de savoir sur quoi portait la réunion de Sienne, il y a deux sortes de réponses. D'une part, on pourrait décrire et analyser les thèmes, les contributions écrites et les débats; d'autre part, l'événement en tant que phénomène semble mériter en soi un commentaire socio-musicologique. En tant qu'observateur-participant, je devrais formuler différemment la question concernant la seconde réponse possible, soit: "Que signifiait être présent?" J'y reviendrai. Essayons tout d'abord d'en résumer le contenu, en évitant de dresser simplement une liste interminable.

Les thèmes proposés pour des communications écrites étaient: «Musique, religion et thérapie» et «Concepts de mode et de genre dans la recherche ethnomusicologique récente». Il y avait, en outre, deux tables rondes avec des participants invités, portant respectivement sur les «Racines de l'ethnomusicologie européenne" et les "Publications ethnomusicologiques en Europe», faisant ainsi référence aux deux thèmes interdépendants de l'histoire de l'ethnomusicologie européenne et de l'histoire européenne de l'ethnomusicologie. En outre, une réunion consacrée aux affaires courantes eut lieu sous la présidence de John Blacking, qui débattit de l'avenir du SEEM. Diego Carpitella et Tràn Van Khê furent élus par acclamation membres d'honneur à vie du SEEM. Trente-quatre contributions écrites furent présentées, tandis que trois interventions étaient largement improvisées (et, de ce fait, suivies avec un intérêt accru). Les intervenants disposaient de vingt minutes chacun, plus dix minutes pour la discussion, du moins selon l'horaire prévu. Les quarante-cinq participants inscrits avaient tous la possibilité de prendre part à l'ensemble des séances grâce à l'échelonnement de la conférence sur trois jours et demi. 
Lors de la table ronde d'ouverture consacrée à l'histoire de l'ethnomusicologie européenne, les communications d'Anna Czekanowska (Varsovie) et d'Izalii Zemtovsky (Leningrad) contenaient une foule de renseignements nouveaux, puisés dans des sources slaves et d'autres régions d'Europe de l'Est. Elles firent état d'une profondeur historique étonnante. Simha Arom (Paris), président de la séance, et Bernard Lortat-Jacob (Paris) intervinrent tous deux pour faire remarquer qu'à leurs yeux, les recherches menées conjointement par des musicologues et des sociologues au milieu des années 1920 en divers pays par diverses équipes constituaient une véritable anthropologie de la musique qui était quarante ans en avance sur celle des États-Unis. Si, un jour, ce point de vue est rendu public en anglais en un lieu approprié, il y aura sans doute une réaction, et c'est tant mieux! J'ai apprécié que le récit historique des travaux menés par les chercheurs soviétiques et autres depuis les années 1880 ait contribué à rétablir l'équilibre perturbé par les prétentions à l'originalité de la science ethnomusicologique nord-américaine (cf. Hood 1989), qui pourrait d'ailleurs résulter d'une méconnaissance de la littérature écrite dans des langues différentes de celles que l'on a coutume de lire.

Tout cela touche un point sensible, et l'une des raisons de la fondation du SEEM: le désir d'échapper à une certaine hégémonie (perçue) de l'Amérique du Nord. La réaffirmation de l'identité de l'ethnomusicologie européenne à l'échelle mondiale est opportune et, à mon sens, elle survient au moment où une bonne partie de la recherche nord-américaine s'essouffle. Selon Gilbert Rouget (cf. Borel 1988: 177-86), le recours au travail de terrain dans un contexte «urbain» revient à renoncer à croire en la nécessité d'une étude urgente de la musique des sociétés pré-industrielles ${ }^{2}$.

Si un historique plus complet de l'ethnomusicologie de l'Europe de l'Est était une nouveauté pour de nombreux participants, la réévaluation qu'elle suscita était aussi due à la contribution italienne à la table ronde ${ }^{3}$. Pietro Clemente (Sienne) adopta une position formaliste en prétendant que l'ethnomusicologie italienne émergea seulement lorsque ses représentants s'approprièrent l'étiquette etnomusicologia et l'utilisèrent de manière autonome (dans les an-

2 Rouget a peut-être raison, mais je pense que d'autres facteurs entrent également en ligne de compte: les réductions budgétaires, faisant qu'il y a moins de fonds pour envoyer les étudiants dans des contrées lointaines; une nouvelle conscience des ressources non exploitées «chez soi »; le protectionnisme pratiqué par une génération de professeurs sur les régions déjà «colonisées» (par l'ethnomusicologie); l'opposition de certaines sociétés (par exemple en Océanie) à la présence de chercheurs par fierté nationale; et l'élévation du niveau de qualification des étudiants en vue de leur initiation au travail de terrain, afin de mieux les préparer au choc ou au «baptême du feu » que leurs enseignants ont déjà subi dans des cultures totalement étrangères, grâce à un travail de terrain mené avec un pied dans chaque camp, si l'on peut dire. Il faut également considérer l'effet débilitant de séances répétées d'introspection académique du genre «où allons-nous?», pour laquelle Rouget montre peu de patience.

3 Lors de l'ouverture de la réunion de Sienne, chaque participant reçut un exemplaire d'Ethnomusicologica, volume édité par Diego Carpitella (1989) qui réunit les contributions de divers chercheurs ayant participé, au cours des derniers treize ans, à l'activité des Séminaires internationaux d'ethnomusicologie. 
nées 1950), en dépit du travail réalisé dans les décennies précédentes sous d'autres étiquettes, notamment par Diego Carpitella, Giorgio Nataletti et Roberto Leydi. Peut-être cette idée paraîtrait-elle moins naïve si, dans le passé, la recherche italienne avait davantage été menée en dehors des frontières nationales, voire coloniales (avant la Seconde Guerre mondiale); mais on m'a dit que cela avait été peu fréquent. J'ai eu l'impression que le groupe italien cherchait toujours une identité plus positive et plus clairement définie, comme l'attestent en partie les attitudes des autres disciplines lorsqu'il s'agit de partager le pouvoir (par exemple, le nombre de postes de recherche et d'enseignement à financer) au sein des institutions universitaires italiennes. Or la source de pareille angoisse reste un mystère, si l'on songe à la richesse des terrains régionaux italiens et à la longue histoire des publications italiennes (y compris les phonogrammes); dommage que nombre de travaux risquent de passer inaperçus sur le plan international pour des raisons linguistiques. Conscients de cela, les hôtes italiens intervenaient soit en français, soit en anglais. On remarqua aussi les liens académiques étroits que certains chercheurs italiens avaient noués avec les États-Unis, y compris des publications en «américain». L'idée d'une table ronde consacrée aux «racines» venait de Diego Carpitella.

Quant au thème «Musique, religion et thérapie», il avait été proposé en 1988, lors du congrès de Tuczno (Pologne), par Igor Bogdanov (Moscou) qui (étant lui-même aussi médecin) a effectué plus de cinquante missions de recherche dans les régions les plus reculées de l'Union soviétique septentrionale et orientale, où il a souvent observé des chamanes pratiquant la musique à des fins de guérison. Le thème était cher au président de la première séance, Francesco Giannattasio (Potenza) qui introduisit les douze communications en évoquant, entre autres, le lien entre ethnomusicologie et ethnopsychiatrie. Robert Günther (Cologne), président de la seconde séance, remarqua plus tard lors du débat général, qu'il était difficile d'identifier des lignes de réflexion communes. Le sujet était vaste, et les intervenants avaient adopté une douzaine de positions différentes pour lancer leurs flèches sur un monolithe. Si le thème paraissait vague et son traitement peu concluant, tout cela n'en a pas moins suscité des propositions utiles pour les rencontres futures (par exemple à Berlin en 1990) lorsque Blacking intervint sur la connaissance musicale. Mais en fait, Blacking avait déjà soulevé la question de la cognition musicale avant que ce débat général n'eût lieu, établissant ainsi un lien entre les deux thèmes. J'y reviendrai.

Le second thème, «Concepts de mode et de genre», fut également abordé en deux séances présidées respectivement par Lortat-Jacob et Czekanowska; elles donnèrent lieu chacune à sept communications. Une série de brefs commentaires sur les agencements sonores d'une musique particulière ne sont pas toujours intéressants à écouter: «il vaudrait mieux les lire à la maison», remarqua Arom.

Le moment fort du congrès fut l'intervention conjointe d'Arom et de son collègue parisien Vincent Dehoux au sujet des «Systèmes d'intervalles en Centrafrique. Expérimentations sur le terrain». Leur exposé de la problématique dans les termes mêmes de peuples qui ne verbalisaient pas tous les éléments de 
leur théorie musicale, fut brillant, et l'aspect «théâtralisé» de leur présentation ajoutait à sa qualité. Excellent conférencier, Arom est aussi un acteur qui investit tout l'espace dont il dispose, qui se lance dans l'imitation enjouée, et qui sait surtout transmettre sa réelle passion pour son sujet. Il se livre à une mise en scène qui souligne son sérieux fondamental, tout en faisant apparaître la force qui le propulse toujours plus profondément dans son domaine d'étude. A son côté, Dehoux servait apparemment de faire-valoir; mais lorsqu'il commença à parler, on eut affaire à forte partie. Et Lortat-Jacob, en président complice, les encouragea: ils servaient à tour de rôle dans une sorte de match de tennis. Nous fûmes ravis de cette joute et de la stimulation intellectuelle qu'elle offrit.

Voici tout d'abord le résumé qu'Arom lui-même donna de son sujet: «Nous avons essayé de faire valider les échelles par les Africains eux-mêmes à l'aide du synthétiseur [Yamaha DX7] qui peut modifier les timbres, les échelles, les topologies d'un clavier, et qui permet aux musiciens eux-mêmes de modifier chaque note comme ils l'entendent, à 1,17 cents près ».

Plus tard, il ajouta: «... on n'est plus dans les problèmes de perception, on touche aux problèmes de conception...».

Divers extraits des quelque trente heures d'enregistrement vidéo de l'expérimentation furent présentés, y compris les trente secondes (désormais célèbres) montrant la rencontre initiale au cours de laquelle l'équipe attend nerveusement si oui ou non sa machine extraordinaire sera agréée. Qu'en est-il des réactions locales? Arom cite le quolibet d'un ancien: «Vous avez triché la voix de mes xylophones pour la mettre là-dedans!». La caravane était partie en sachant que les mensurations subtiles effectuées par l'IRCAM sur les échelles (précédemment enregistrées) du xylophone avaient été tenues pour «incohérentes», en termes mathématiques, en tant que système. Je pense que cela veut dire que, statistiquement parlant, la variabilité mesurée en accords a donné plus d'éléments aléatoires que prévu. Mais ce n'était là qu'une seule parmi plusieurs problématiques.

Blacking se leva pour s'adresser à Arom et à son équipe:

«...C'est une expérimentation formidable que vous avez effectuée, c'est un exemple parfait de ce que j'ai appelé l'ethnomusicologie dialectique... Ce sur quoi j'aimerais insister, si je peux, c'est que je tiens à souligner certains aspects que vous avez évoqués, car je pense qu'ils sont fort importants et aussi pertinents au regard du problème général de la cognition musicale. Le premier concerne le problème de la verbalisation et le besoin d'élaborer des tests de ce genre... Je pense que cela devrait être une technique standard dans toutes sortes de recherches ethnomusicologiques... Certes, on peut le faire avec un équipement moins sophistiqué - mais le principe de ce genre de choses devrait rester le même! Par exemple, vous avez attiré l'attention sur la question de savoir "où les notes se situent" sur une échelle ou une rangée supposée. C'est un problème que j'ai rencontré chez les Venda: on avait des chansons enfantines qui avaient le même [il chante] "do" - la - sol - fa" dérivant d'une échelle soit pentatonique, soit heptatonique, mais laquelle des deux?... Toute cette question (...) d'échelles et de contextes "indéterminés" est cruciale...». 
Le restant des communications consacrées aux modes et aux genres ne surprit guère, mais le niveau général de la recherche était mieux assuré que dans le premier thème principal. En annonçant la prochaine réunion à Berlin, Ulrich Wegner fit savoir qu'il souhaitait distribuer au moins un mois à l'avance un document préliminaire contenant les communications retenues, afin que les participants au Séminaire de 1990 puissent consacrer un maximum de temps à la discussion. Il est vrai qu'à Sienne, la succession des interventions a épuisé la capacité de concentration des auditeurs, et ceux qui avaient la malchance de parler en fin de séance étaient inévitablement déçus par le manque de réactions d'une assistance fatiguée. Quant au document préliminaire, c'est en principe une bonne idée, sans doute, à condition que tout le monde fasse ses devoirs... Je serais moi-même plutôt en faveur d'une documentation préliminaire partielle, c'est-à-dire qui contienne les interventions potentiellement les plus difficiles, allant de pair avec d'autres séances plus improvisées afin de réserver un élément de surprise, séances pour lesquelles les participants pourraient avoir une semaine de préparation en vue de rédiger une page d'idées percutantes sur un thème réellement controversé - page à multicopier et à distribuer sur place.

En ce qui me concerne, je serais heureux d'assister à une réunion du SEEM qui mette à disposition un équipement de copie audio-visuelle simple, rapide, peu onéreux et de bonne qualité, afin de faciliter l'échange de matériel parmi les participants. C'est particulièrement important pour les participants venant des pays de l'est, en raison des difficultés qui jalonnent l'échange d'informations - autant que d'argent - entre l'est et l'ouest.

La table ronde consacrée aux publications européennes révéla la nécessité de disposer d'une publication préliminaire ou, sur place, d'un service de multicopie en gros. Les divers rapports contenaient une masse énorme de données que l'on avait de la peine à noter en détail, à cause de l'allure du débat. Comme Ross Clark l'a remarqué ailleurs, le fait de tenir un journal empêche parfois de vivre une expérience cruciale... Quoi qu'il en soit, il se passe beaucoup de choses, y compris quelques initiatives heureuses (comme ces Cahiers). Étant donné que certains rapports étaient présentés sous une forme résumée, l'absence de références aux publications existantes causa parfois quelque irritation. Mireille Helffer remarqua que les disques «ethniques» en France paraissent et s'épuisent de manière si aléatoire qu'il est fort difficile de rester à jour dans ce domaine. Par exemple, plusieurs disques 33 tours publiés par JeanClaude Chabrier (Paris) n'ont pas été mentionnés, bien qu'ils soient toujours disponibles. Le système du "dépôt légal» ne fournit-il pas une source fiable pour connaître les nouvelles parutions?

Quant à la question de savoir ce qu'a été le séjour à Sienne, tout le monde semble avoir été charmé par la ville et par l'accueil chaleureux au séminaire de Montarioso. Ce qu'a signifié la rencontre elle-même ressort, je l'espère, du présent compte rendu. Mais je me suis demandé pourquoi nous n'avons eu droit qu'à un seul concert et pourquoi nous fûmes privés de la possibilité de rencontrer ou d'entendre des interprètes italiens traditionnels? 


\section{Bibliographie}

BOREL François

1988 «Entretien avec Gilbert Rouget». Cahiers de Musiques Traditionnelles 1: 177-86.

CARPITELLA Diego (ed.)

1989 Ethnomusicologica. Seminari Internazionali di Siena 1977-1989. Quaderni dell'Accademia Musicale Chigiana XIIII (rédacteurs: G. Burchi \& G. Giurati). Siena: Accademia Musicale Chigiana.

\section{HOOD Ki Mantle}

1989 «Music from Galileo to Einstein : a quantum leap». Progress Reports in Ethnomusicology 2(8) : passim.

\section{PHILIPP Margot Leith (ed.)}

1989 Ethnomusicology and the Historical Dimension. (Papers presented at the European Seminar in Ethnomusicology, London, 20-23 May 1986). Ludwigsburg: Philipp Verlag, 144 p. 